\title{
Validade Preliminar da Escala Perfil do Estilo de Vida Individual para uso online em tempos de distanciamento social
}

\author{
Preliminary validity of Individual Lifestyle Profile Scale for online use in times of \\ social distancing
}

\section{AUTORES \\ Sueyla Ferreira da Silva dos $\operatorname{Santos}^{1}$ (DD \\ Thiago Ferreira de Sousa ${ }^{2}$ (D) \\ Silvio Aparecido Fonseca ${ }^{3}$ (D) \\ Mauro Virgílio Gomes de Barros ${ }^{4}$ (ID \\ Gildeene Silva Farias ${ }^{5}$ (D) \\ Markus Vinicius Nahas ${ }^{6}$ (D) \\ 1 Universidade Federal do Amazonas, Instituto de Ciências Sociais, Educação e Zootecnia, Parintins, Amazonas, Brasil. \\ 2 Universidade Federal do Recôncavo da Bahia, Centro de Formação de Professores, Amargosa, Bahia, Brasil. \\ 3 Universidade Estadual de Santa Cruz, \\ Departamento de Saúde, Ilhéus, Bahia, Brasil. \\ 4 Universidade de Pernambuco, Departamento de \\ Educação Física, Recife, Pernambuco, Brasil. \\ 5 Faculdade Estácio de Teresina, Departamento de Educação Física, Teresina, Piauí \\ 6 Universidade Federal de Santa Catarina, Departamento de Educação Física, Florianópolis, Santa Catarina, Brasil.}

\section{CONTATO}

Sueyla Ferreira da Silva dos Santos sueylasantos@ufam.edu.br

Instituto de Ciências Sociais, Educação e Zootecnia (ICSEZ/UFAM). Estrada Parintins/Macurany, 1805, bairro Jacareacanga, Parintins, Amazonas

CEP: 69152-240.

DOI

$10.12820 /$ rbafs.26e 0210

\section{(cc) BY}

Este trabalho está licenciado com uma Licença Creative Commons - Atribuição 4.0 Internacional.

\begin{abstract}
RESUMO
Este estudo teve como objetivo estimar a validade de conteúdo e o nível de reprodutibilidade da escala "Perfil do Estilo de Vida Individual" em tempos de distanciamento social. Os itens da escala original proposta por Nahas, Barros e Francalacci, foram modificados para avaliar o estilo de vida de adultos, de forma online, durante o período distanciamento social, a exemplo, a pandemia da COVID-19. As 15 questões contemplaram comportamentos recomendados por especialistas em saúde durante a pandemia, mantendo-se os cinco domínios originais propostos na versão inicial: alimentação saudável, atividade física, controle do estresse, relacionamentos e comportamentos preventivos. Foram avaliadas a adequação e pertinência por meio do julgamento de especialistas ( $\mathrm{n}$ = 38) e, posteriormente, os profissionais e estudantes $(n=71)$ de Instituições de Ensino Superior (IES) avaliaram a clareza dos itens. A reprodutibilidade foi estimada via coeficiente Kappa. O nível de significância adotado foi de 5\%. A validade de conteúdo mostrou valores médios de adequação, pertinência e clareza de $92,4 \%, 87,1 \%$ e $78,7 \%$, respectivamente. Participaram na etapa de reprodutibilidade 83 estudantes e profissionais de IES e o nível de concordância variou de 0,358 referente ao item "G" (Procuro ocupar a maior parte do meu dia com atividades interessantes) a 0,626 relativo ao item "N" (Mantenho o distanciamento físico e uso máscara sempre que preciso sair). Conclui-se que a escala "Perfil do Estilo de Vida Individual" em tempos de distanciamento social apresentou satisfatória validade de conteúdo e os níveis de reprodutibilidade podem ser considerados aceitáveis para escalas desta natureza.
\end{abstract}

Palavras-chave: Reprodutibilidade dos testes; Estilo de vida; Adultos; Isolamento Social; Pandemia; COVID-19.

\section{ABSTRACT}

This study aimed to estimate the content validation and the reproducibility level of the "Individual Lifestyle Profile" scale in times of social distancing. The items on the scale originally proposed by Nahas, Barros and Francalacci, were modified to assess the lifestyle of adults, of online form, during social distancing period, for example the COVID-19 pandemic. The 15 questions covered the behavior considered by health experts during a pandemic, maintaining the original five domains proposed in the initial version: healthy eating, physical activity, stress control, preventive behavior and relationships. Adequacy and pertinence were assessed through the judgment of specialists $(n=38)$ and, subsequently, professionals and students $(n=71)$ from Higher Education Institutions (HEIs) assess the clarity of the items. Reproducibility was estimated using the Kappa coefficient. The level of significance adopted was 5\%. The average of the validity content of adequacy, relevance and clarity was $92.4 \%, 87.1 \%$ and $78.7 \%$, respectively. 83 students and professionals of HEI participated in the reproducibility stage and the level of agreement ranged from 0.358 for item " $G$ " (I try to occupy most of my day with interesting activities) to 0.626 for item " $N$ " (I keep my physical distance and wear a mask whenever I need to leave). Concluded that "Individual Lifestyle Profile" scale in times of social distancing presented satisfactory content validity and reproducibility levels may be acceptable for scales of this nature.

Keywords: Reproducibility of tests; Lifestyle; Adults; Social isolation; Pandemic; COVID-19.

\section{Introdução}

A pandemia da COVID-19 representa um problema de saúde pública de magnitude elevada ${ }^{1}$, que afetou diversos setores da sociedade e o modo como as pessoas se relacionam, as crenças e valores subjetivos associados aos eventos de cura e tratamento da doença ${ }^{2}$. Além dos problemas de saúde relacionados ao prognóstico da doença, com a possibilidade de morte, a COVID-19 
afetou diretamente o estilo de vida das pessoas, devido as medidas sanitárias necessárias para minimizar o risco de transmissão do vírus ${ }^{3}$.

Características como solidão, sedentarismo e uso de substâncias psicoativas (álcool e outras drogas) foram fatores presentes no cotidiano da população adulta durante a pandemia ${ }^{3,4}$. Estudo de revisão integrativa mostrou que a privação da convivência social, ocasionada pelas medidas restritivas, pode ter repercutido no surgimento de sintomas psíquicos como estresse, ansiedade e depressão na população durante a pandemia da COVID-195.

Diversos grupos de pesquisa direcionaram esforços enfatizando a pandemia da COVID-196 ${ }^{6}$, assim como ocorreu o aumento no uso de websurveys como importante estratégia para realização de estudos primários nas diferentes áreas de conhecimento, inclusive nas Ciências da Saúde ${ }^{7,8}$. Entende-se como websurveys, as estratégias de coleta de dados por meio de links de acesso a formulários online ou utilizando-se de cadastros prévios de endereços eletrônicos e painéis de usuários de internet da população investigada ${ }^{8}$. Torna-se essencial o emprego de medidas psicométricas adequadas, para fins de mensuração de características fidedignas e que atendam aos preceitos éticos e de segurança dos dados digitais na aplicação de questionários online ${ }^{8}$.

Diante desta demanda, a escala "Perfil do Estilo de Vida", fundamentada no modelo do Pentáculo do Bem-Estar, revelou-se robusta interpretação da escala, baseado na teoria de resposta ao item ${ }^{9}$, com nível de variância explicada de $58,6 \%{ }^{10}$ e nível de concordância entre $74 \%$ e $93 \%$ nos diferentes domínios ${ }^{11}$. O referido instrumento avalia as características que afetam a saúde e o bem-estar de adultos, sejam mais jovens, de meia-idade ou professores, e considera cinco componentes do estilo de vida, a saber: atividade física, nutrição, controle do estresse, relacionamentos e comportamentos preventivos. Todavia, esta versão não reflete o contexto social durante a pandemia da COVID-19 e não foi adaptada para a aplicação em formato digital.

Sendo assim, tendo a necessidade da aplicação de um questionário online específico para avaliar o estilo de vida de adultos durante o período de distanciamento social por consequência da pandemia COVID-19, recorreu-se a adaptação dessa escala. Ressalta-se que, o uso de um modelo conceitual ${ }^{12}$, juntamente com estratégias que contemplem adaptações de questões operacionais e psicométricas ${ }^{13}$, podem viabilizar o uso desta escala em estudos epidemiológicos, principalmente para fins educacionais. Em função disso, o objetivo deste estudo foi estimar a validade de conteúdo e do nível de reprodutibilidade da escala "Perfil do Estilo de Vida Individual" em tempos de distanciamento social, a exemplo, a pandemia da COVID-19.

\section{Métodos}

As informações deste estudo fazem parte da pesquisa "Impacto da pandemia COVID-19 no estilo de vida de discentes e servidores de instituições de ensino superior", aprovado pelos comitês de éticas em pesquisa com seres humanos das instituições: Universidade Federal do Recôncavo da Bahia (UFRB), CAEE 33377120.0.0000.0056; Universidade Federal do Sul da Bahia (UFSB), CAEE 33377120.0.3010.8467; Universidade Federal da Bahia (UFBA), CAEE 33377120.0.3005.5531; Universidade Estadual de Santa Cruz (UESC), CAEE 33377120.0.3007.5526; Universidade Federal do Amazonas (UFAM), CAEE 33377120.0.3002.5020; Universidade Federal do Alagoas (UFAL), CAEE 33377120.0.3008.5013; Universidade Federal do Triangulo Mineiro (UFTM), CAEE 33377120.0.3006.5154; Universidade Federal de Viçosa (UFV), CAEE 33377120.0.3003.5153; Universidade Federal do Mato Grosso do Sul (UFMS), 33377120.0.3001.0021; Universidade Federal de Santa Maria (UFSM), CAEE 33377120.0.3004.5346 e Centro Universitário União de Ensino do Sudoeste do Paraná (UNISEP), CAEE: 33377120.0.3009.5230.

Este estudo é referente a etapa de validade dos instrumentos de pesquisa e foi constituído de quatro fases (Figura 1). A primeira, corresponde a elaboração do instrumento com base o referencial teórico, que considera o estilo de vida como as "ações habituais que refletem as atitudes, os valores e as oportunidades na vida das pessoas"11,14. A segunda, refere-se a análise de pertinência e adequação; terceira sobre aplicação do instrumento para a identificação do nível de clareza; por fim, a quarta fase, mediante a análise de reprodutibilidade.

Para a elaboração do instrumento adotou-se as perguntas referentes a escala "Perfil do Estilo de Vida Individual", versão adultos ${ }^{11}$, validado para a população de brasileiros ${ }^{10}$. $\mathrm{O}$ instrumento adaptado seguiu o padrão da versão original, sendo composto por 15 questões que permitem a avaliação do estilo de vida em relação as cinco dimensões: alimentação saudável, atividade física, controle do estresse, relacionamentos e comportamento preventivos.

Para cada item a pessoa é orientada a indicar a afir- 


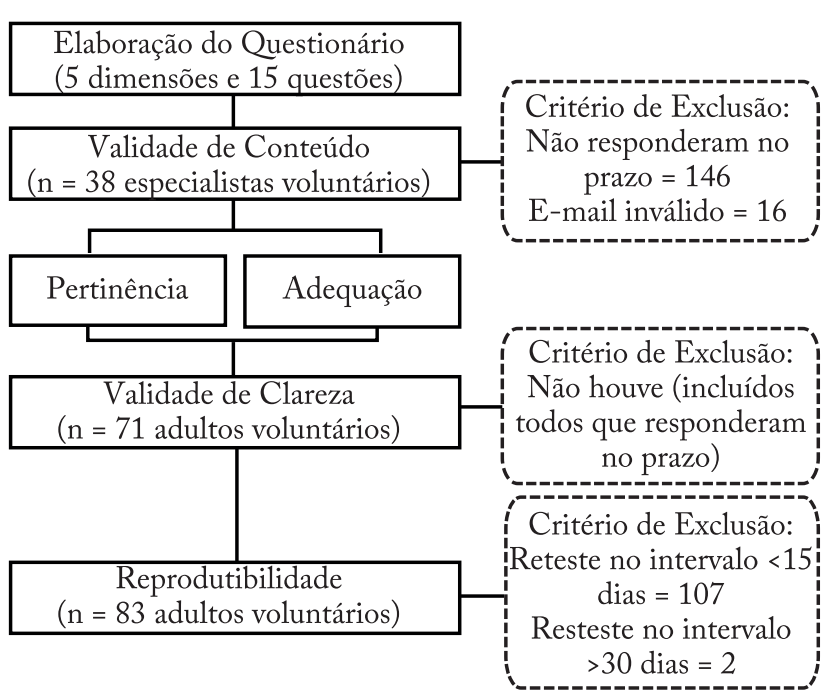

Figura 1 - Etapas de validação preliminar da escala "Perfil do Estilo de Vida Individual" em tempos de distanciamento social.

mação que melhor corresponde ao seu estilo de vida atual. As opções de resposta foram elaboradas seguindo o mesmo padrão do instrumento prévio, em uma escala de Likert de quatro pontos: (0) absolutamente não faz parte do seu estilo de vida; (1) às vezes corresponde ao seu comportamento; (2) quase sempre verdadeiro no seu comportamento; (3) a afirmação é sempre verdadeira no seu dia-a-dia; faz parte do seu estilo de vida.

A versão inicial do instrumento foi submetida a validação de conteúdo e, posteriormente de clareza. $\mathrm{Na}$ etapa de validação de conteúdo, foram convidados por conveniência, via e-mail de contato disponibilizado no currículo digital dos pesquisadores, entre os dias 20 de junho e 4 de julho de 2020, 38 especialistas da área da saúde com expertise em comportamento humano, estilo de vida e saúde pública. Os especialistas foram selecionados mediante a consulta do currículo de pesquisadores de Instituições de Ensino Superior (IES) e membros de sociedades científicas na área da saúde e da atividade física e saúde. Os convidados que aceitaram participar foram orientados a avaliar o enunciado do questionário; a pertinência das perguntas e a adequação das escalas de resposta, bem como acrescentar sugestões gerais que considerassem pertinentes para avaliação do instrumento.

Para avaliação da pertinência foi apresentada a seguinte pergunta: "A pergunta [número da pergunta] é pertinente a dimensão [nome da dimensão]?", sendo as opções de resposta "sim" e "não". Considerou-se, como critério positivo e aceitável de pertinência a resposta "sim". Para avaliação da adequação foi apresentada a seguinte pergunta: "Em relação a pergunta [número da pergunta], as opções de resposta estão?”, sendo as opções de resposta, a partir de uma escala de Likert de cinco pontos: (1) muito inadequadas; (2) inadequadas; (3) nem adequadas, nem inadequadas; (4) adequadas; e, (5) muito adequadas. Considerou-se, como critério positivo e aceitável de adequação, a junção das opções (4) adequadas e (5) muito adequadas.

Para análise de clareza foram convidados por conveniência, via mídias sociais do projeto de pesquisa e grupos de IES brasileiras, com exceção de membros da comunidade universitária que seriam convidados para a etapa seguinte (reprodutibilidade), entre os dias $13 \mathrm{e}$ 23 de julho de 2020, 71 voluntários (docentes, técnicos e discentes) para o preenchimento de um painel online para que respondessem a seguinte pergunta: "Na sua opinião, em que medida a afirmativa e respectivas opções de resposta estão claras ou confusas?". Para cada item do questionário foram dadas as seguintes opções de resposta, a partir de uma escala de Likert de cinco pontos: (1) muito confusa; (2) confusa; (3) nem confusa, nem clara; (4) clara; e, (5) muito clara. Considerou-se, como critério positivo e aceitável de clareza, a junção das opções (4) clara e (5) muito clara.

Para a última etapa, voluntários selecionados por conveniência participaram dessa etapa do estudo em dois momentos (teste e reteste). Os convites para a participação ocorreram por meio de correspondência eletrônica e nas redes sociais das IES. Foram convidados servidores (docentes e técnicos) e estudantes de graduação de 11 IES brasileiras, a saber: UFRB, UFSB, UFBA, UESC, UFAM, UFAL, UFTM, UFV, UFMS, Faculdade Metodista de Santa Maria (FSM) e UNISEP.

Foram incluídos os participantes que apresentaram vínculo institucional ativo com as instituições supracitadas e que aceitaram participar, via anuência por meio do Termo de Consentimento Livre e Esclarecido (TCLE). Foram considerados para análise de reprodutibilidade o instrumento respondido em duas ocasiões, com diferença de 15 a 30 dias. Estimou-se como amostra mínima 55 pessoas, tendo sido considerado 58,65\% de explicação do instrumento original ${ }^{10}$, poder de $80 \%$ e nível de confiança de $95 \%$. O tamanho da amostra foi calculado no software BioEstat, versão 5.3.

Todos os formulários foram elaborados na plataforma Google Forms e as respostas obtidas foram automaticamente armazenadas em planilha do Excel para fins das análises relativas à pertinência, adequação e clareza. Adotou-se como valores aceitáveis de pertinência, adequação e clareza, frequências relativas maiores ou iguais 
a $70 \%{ }^{15}$ por item, por domínio e no escore total. $\mathrm{Na}$ etapa de formulação do instrumento, decidiu-se que as fases de adequação e pertinência realizada por especialistas, teriam o peso maior na decisão de alterações do instrumento.

Para a etapa de reprodutibilidade, as análises foram realizadas no software SPSS, versão 24.0, empregou-se análises descritivas de média e desvio padrão para a variável idade e frequências absolutas e relativas para as variáveis de vínculo com a instituição de ensino superior, e para estimar a concordância foi utilizado o índice $K a p p a(\mathrm{k})$, com a seguinte classificação: $\leq 0,20=$ concordância leve, pobre; 0,21 a $0,40=$ concordância fraca, regular; 0,41 a $0,60=$ concordância moderada; 0,61 a $0,80=$ concordância forte; $\geq 0,80=$ concordância perfeita $^{16}$. O nível de significância adotado foi de $5 \%$.

\section{Resultados}

O painel de validade de conteúdo foi composto por 38 especialistas (60,5\% doutores) que foram registradas numa plataforma de resposta online. Os valores percentuais das análises de pertinência e adequação são apresentados na Tabela 1 . Dados gerais revelaram valores positivos de pertinência $(92,4 \%)$ das perguntas em relação as dimensões e, em relação a adequação $(87,1 \%)$ das opções de resposta para a pergunta.

Baseado nas sugestões apresentadas, foi considerado relevante realizar ajustes no instrumento quanto à organização das perguntas e redação das questões, especialmente a inclusão de itens que tratassem sobre evitar o consumo excessivo de álcool e a prática do deslocamento ativo. $\mathrm{O}$ instrumento foi ajustado quanto à inclusão de termos que indicassem temporalidade, a saber "muito tempo sentado", "mais ou menos regulares" e "maior parte".

Após as modificações, o instrumento foi submetido ao procedimento de validade de clareza. Houve a participação de 71 voluntários (41 docentes, 5 técnicos administrativos e 25 discentes de graduação) de IES públicas (federais: 23,1\%; estaduais: 43,1\%) e privadas $(33,8 \%)$ na etapa da clareza, mediante o preenchimento do formulário online. A validade de clareza média do instrumento foi de $78,7 \%$, e a maioria das questões (53\%) apresentaram níveis de clareza superior à média alcançada. $\mathrm{Na}$ Tabela 2 são apresentados os valores percentuais de clareza.

No Quadro 1 é apresentada a versão final do instrumento, juntamente com o enunciado e orientações para o preenchimento. $\mathrm{Na}$ etapa de reprodutibilidade
Tabela 1 - Valores percentuais das análises de pertinência e adequação da escala "Perfil do Estilo de Vida Individual" em tempos de distanciamento social, 2020.

\begin{tabular}{|c|c|c|c|}
\hline Domínio & Item & $\begin{array}{l}\% \\
\text { Pert. }\end{array}$ & $\begin{array}{c}\% \\
\text { Adeq. }\end{array}$ \\
\hline \multicolumn{2}{|c|}{ Alimentação Saudável } & 97,4 & 89,4 \\
\hline & $\begin{array}{l}\text { A. Faço } 3 \text { ou } 4 \text { refeições leves ao } \\
\text { dia, em horários definidos. }\end{array}$ & 94,9 & 94,8 \\
\hline & $\begin{array}{l}\text { B. Minhas refeições incluem frutas, } \\
\text { hortaliças e cereais integrais. }\end{array}$ & 100,0 & 92,3 \\
\hline & $\begin{array}{l}\text { C. Evito alimentos gordurosos, } \\
\text { doces e alimentos industrializados. }\end{array}$ & 97,4 & 81,1 \\
\hline \multirow[t]{4}{*}{ Atividade Física } & & 83,7 & 82,9 \\
\hline & $\begin{array}{l}\text { D. Faço intervalos e me movimento } \\
\text { (10 minutos a cada hora) quando } \\
\text { passo muito tempo sentado. }\end{array}$ & 92,3 & 89,7 \\
\hline & $\begin{array}{l}\text { E. Tenho uma rotina de exercícios } \\
\text { simples (ginástica, alongamentos, } \\
\text { yoga, etc.). }\end{array}$ & 97,4 & 89,7 \\
\hline & $\begin{array}{l}\text { F. Aprecio dançar ou assistir vídeos } \\
\text { de jogos ou recreação ativos. }\end{array}$ & 61,5 & 69,2 \\
\hline \multicolumn{2}{|c|}{ Controle do Estresse } & 92,9 & 87,2 \\
\hline & $\begin{array}{l}\text { G. Procuro me ocupar a maior parte } \\
\text { do dia com atividades interessantes. }\end{array}$ & 87,2 & 82,1 \\
\hline & $\begin{array}{l}\text { H. Procuro contatar, mesmo que } \\
\text { virtualmente, pessoas amigas } \\
\text { sempre que me sinto muito ansioso } \\
\text { ou só. }\end{array}$ & 94,2 & 89,7 \\
\hline & $\begin{array}{l}\text { I. Reservo alguns momentos diários } \\
\text { para relaxar, ler, ouvir, música ou } \\
\text { meditar. }\end{array}$ & 97,4 & 89,8 \\
\hline \multirow{4}{*}{ Relacionamentos } & & 90,6 & 86,3 \\
\hline & $\begin{array}{l}\text { J. Mantenho contatos frequentes, } \\
\text { mesmo que virtualmente, com } \\
\text { amigos e parentes próximos. }\end{array}$ & 92,3 & 92,3 \\
\hline & $\begin{array}{l}\text { K. Procuro me conhecer cada vez } \\
\text { mais, valorizando a espiritualidade } \\
\text { ou autoconhecimento. }\end{array}$ & 82,1 & 79,5 \\
\hline & $\begin{array}{l}\text { L. Mesmo em isolamento social, } \\
\text { procuro me manter útil na } \\
\text { comunidade. }\end{array}$ & 97,4 & 87,2 \\
\hline \multicolumn{2}{|c|}{ Comportamentos Preventivos } & 97,4 & 89,6 \\
\hline & $\begin{array}{l}\text { M. Lavo as mãos com frequência e } \\
\text { faço uso de álcool gel para as mãos } \\
\text { e objetos de uso cotidiano. }\end{array}$ & 100,0 & 89,7 \\
\hline & $\begin{array}{l}\text { N. Mantenho o distanciamento } \\
\text { físico e uso máscara sempre que } \\
\text { preciso sair. }\end{array}$ & 97,4 & 89,8 \\
\hline & $\begin{array}{l}\text { O. Após realizar compras, higienizo } \\
\text { os produtos e deixo calçados fora } \\
\text { de casa. }\end{array}$ & 94,9 & 89,2 \\
\hline Escore total & & 92,4 & 87,1 \\
\hline
\end{tabular}

houve a participação de 192 servidores e discentes. No entanto, 107 participaram no segundo momento com menos de 15 dias e dois com mais de 30 dias. Por fim, a amostra final foi de 83 voluntários, sendo 3 da UFRB, 
Tabela 2 - Valores percentuais da análise de clareza da escala "Perfil do Estilo de Vida Individual" em tempos de distanciamento social, 2020.

\begin{tabular}{|c|c|c|}
\hline Domínio & Item & \% Clareza \\
\hline \multicolumn{2}{|c|}{ Alimentação Saudável } & 78,4 \\
\hline & $\begin{array}{l}\text { A. Faço } 3 \text { ou } 4 \text { refeições ao dia, em } \\
\text { horários mais ou menos regulares. }\end{array}$ & 71,8 \\
\hline & $\begin{array}{l}\text { B. Incluo frutas, verduras e legumes em } \\
\text { minhas refeições. }\end{array}$ & 85,9 \\
\hline & $\begin{array}{l}\text { C. Evito incluir nas minhas refeições } \\
\text { alimentos gordurosos e doces. }\end{array}$ & 77,4 \\
\hline \multirow[t]{4}{*}{ Atividade Física } & & 73,7 \\
\hline & $\begin{array}{l}\text { D. Faço intervalos e me movimento } \\
\text { quando passo muito tempo sentado. }\end{array}$ & 67,6 \\
\hline & $\begin{array}{l}\text { E. Tenho uma rotina diária que } \\
\text { inclui atividades físicas (ginástica, } \\
\text { alongamentos, ioga, subir escadas etc.). }\end{array}$ & 78,8 \\
\hline & $\begin{array}{l}\text { F. Caminho ou pedalo, mantendo } \\
\text { distanciamento seguro, quando preciso ir } \\
\text { a lugares próximos. }\end{array}$ & 74,6 \\
\hline \multicolumn{2}{|c|}{ Controle do Estresse } & 75,1 \\
\hline & $\begin{array}{l}\text { G. Procuro ocupar a maior parte do meu } \\
\text { dia com atividades interessantes. }\end{array}$ & 66,2 \\
\hline & $\begin{array}{l}\text { H. Quando me sinto só ou ansioso } \\
\text { procuro ajuda de familiares e amigos, } \\
\text { mesmo que virtualmente. }\end{array}$ & 76,1 \\
\hline & $\begin{array}{l}\text { I. Reservo alguns momentos do meu dia } \\
\text { para relaxar, ler, ouvir música ou meditar. }\end{array}$ & 83,1 \\
\hline \multirow[t]{4}{*}{ Relacionamentos } & & 78,9 \\
\hline & $\begin{array}{l}\text { J. Mantenho contatos/conversas } \\
\text { frequentes, mesmo que virtualmente, com } \\
\text { amigos e parentes próximos. }\end{array}$ & 87,3 \\
\hline & $\begin{array}{l}\text { K. Procuro me conhecer cada vez } \\
\text { mais, valorizando a espiritualidade/ } \\
\text { religiosidade. }\end{array}$ & 81,7 \\
\hline & $\begin{array}{l}\text { L. Mesmo em isolamento, procuro me } \\
\text { manter útil na comunidade. }\end{array}$ & 67,6 \\
\hline \multicolumn{2}{|c|}{ Comportamentos Preventivos } & 87,4 \\
\hline & $\begin{array}{l}\text { M. Lavo as mãos com frequência e faço } \\
\text { uso de álcool gel para mãos e objetos de } \\
\text { uso cotidiano. }\end{array}$ & 85,9 \\
\hline & $\begin{array}{l}\text { N. Mantenho o distanciamento físico e } \\
\text { uso máscara sempre que preciso sair. }\end{array}$ & 90,2 \\
\hline & $\begin{array}{l}\text { O. Evito o consumo exagerado de bebidas } \\
\text { alcoólicas. }\end{array}$ & 86,0 \\
\hline Escore total & & 78,7 \\
\hline
\end{tabular}

1 da UFSB, 37 da UFBA, 7 da UESC, 12 da UFAM, 6 da UFAL; 4 da UFTM, 7 da UFV, e 6 da UFMS. Não houve participantes da FSM e da UNISEP. Participaram $65,1 \%(n=54)$ estudantes, $12 \%(n=10)$ técnicos e $22,9 \%(n=19)$ docentes. As médias de idade no teste e reteste foram de $33,06(\mathrm{DP}=12,54)$ e $33,12(\mathrm{DP}=$ 12,51) anos, respectivamente.
As informações sobre o nível de concordância são apresentadas na Tabela 3. Os coeficientes de concordância $(\mathrm{k})$ variaram de 0,358 para o item "G" "Procuro ocupar a maior parte do meu dia com atividades interessantes" a 0,626 para o item "N" "Mantenho o distanciamento físico e uso máscara sempre que preciso sair”.

Tabela 3 - Nível de concordância dos itens de reprodutibilidade da escala "Perfil do Estilo de Vida Individual" em tempos de distanciamento social, 2020.

\begin{tabular}{lcccc}
\hline Domínio & Itens & Kappa & Concordância & $\mathrm{p}$ \\
\hline \multirow{3}{*}{ Alimentação Saudável } & A & 0,525 & Moderada & $<0,001$ \\
& B & 0,450 & Moderada & $<0,001$ \\
& C & 0,416 & Moderada & $<0,001$ \\
Atividade Física & D & 0,497 & Moderada & $<0,001$ \\
& E & 0,531 & Moderada & $<0,001$ \\
Controle do Estresse & F & 0,438 & Moderada & $<0,001$ \\
& G & 0,358 & Fraca/Regular & $<0,001$ \\
& H & 0,381 & Fraca/Regular & $<0,001$ \\
Relacionamentos & I & 0,372 & Fraca/Regular & $<0,001$ \\
& J & 0,386 & Fraca/Regular & $<0,001$ \\
& K & 0,471 & Moderada & $<0,001$ \\
Comportamentos & L & 0,411 & Moderada & $<0,001$ \\
Preventivos & M & 0,547 & Moderada & $<0,001$ \\
& N & 0,626 & Forte & $<0,001$ \\
& O & 0,554 & Moderada & $<0,001$ \\
\hline
\end{tabular}

\section{Discussão}

O estudo objetivou estimar a validade de conteúdo e o nível de reprodutibilidade da escala "Perfil do Estilo de Vida Individual" em tempos de distanciamento social. Os resultados apresentaram níveis satisfatórios de adequação, pertinência, clareza para utilização deste instrumento na avaliação do estilo de vida (individual ou de grupos) e nas intervenções para promover estilos de vida saudáveis na população adulta.

A validade de conteúdo, que compreende etapa de análise do instrumento com base o referencial teórico ou com a apreciação de especialistas de determinada área, representa uma estratégia mínima para verificação da qualidade das medidas do tipo papel e caneta que serão empregadas em um estudo ${ }^{17}$. Valores aceitáveis para a validade de conteúdo podem ser descritos como no mínimo $80 \%{ }^{17}$. Neste estudo os níveis de adequação e pertinência, pertencentes a validade de conteúdo, alcançaram valores médios de $92,4 \%$ e $87,1 \%$, respectivamente, o que demonstra valores satisfatórios do instrumento, corroborando com outro estudo ${ }^{18}$ que estimou validade de conteúdo em 95,7\% para um questionário destinado a mensuração de características relacionadas 
Quadro 1 - Apresentação da escala "Perfil do Estilo de Vida Individual” em tempos de distanciamento social, na sua última versão, 2020.

\section{PERFIL DO ESTILO DE VIDA INDIVIDUAL - EMTEMPOS DE} DISTANCIAMENTO SOCIAL

O ESTILO DE VIDA corresponde ao conjunto de ações habituais que refletem as atitudes, valores e oportunidades na vida das pessoas. Essas ações têm grande influência na saúde geral e na qualidade de vida de todos os indivíduos.

Durante o necessário período de isolamento em casa, precisamos manter certas rotinas promotoras de saúde (física e mental), diminuindo as chances de nos tornarmos muito sedentários, de agravarmos as situações de estresse e de ganharmos peso.

O primeiro passo para melhorarmos nossos hábitos neste período é avaliar como estamos nos comportando, considerando as cinco dimensões do

Pentáculo do Bem-estar, um modelo proposto para educar e motivar as pessoas para terem estilos de vida mais ativos e saudáveis.

Leia as afirmações abaixo e marque o número que você acha que corresponde ao seu jeito de viver atual.

Escala:

[ 0 ] absolutamente não faz parte do seu estilo de vida

[ 1 ] às vezes corresponde ao seu comportamento

[2] quase sempre verdadeiro no seu comportamento

[ 3 ] a afirmação é sempre verdadeira no seu dia-a-dia; faz parte do seu estilo de vida

\section{Alimentação saudável}

A. Faço 3 ou 4 refeições ao dia, em horários mais ou menos regulares.

1

B. Incluo frutas, verduras e legumes em minhas refeições.

0

1

2

C. Evito incluir nas minhas refeições alimentos gordurosos e doces.

0

\section{Atividade Física}

D. Faço intervalos e me movimento quando passo muito tempo sentado.$$
2
$$$$
3
$$

E. Tenho uma rotina diária que inclui atividades físicas (ginástica, alongamentos, ioga, subir escadas etc.).$$
0
$$$$
1
$$

F. Caminho ou pedalo, mantendo distanciamento seguro, quando preciso ir a lugares próximos.$$
0
$$$$
1
$$$$
2
$$

(1)

\section{Controle do Estresse}

G. Procuro ocupar a maior parte do meu dia com atividades interessantes.

0

1

3

H. Quando me sinto só ou ansioso procuro ajuda de familiares e amigos, mesmo que virtualmente.

0

1

3

I. Reservo alguns momentos do meu dia para relaxar, ler, ouvir música ou meditar.

0

1

2

3

\section{Relacionamentos}

J. Mantenho contatos/conversas frequentes, mesmo que virtualmente, com amigos e parentes próximos.

0

1

2

3

K. Procuro me conhecer cada vez mais, valorizando a espiritualidade/ religiosidade.

0

1

2

3

L. Mesmo em isolamento, procuro me manter útil na comunidade.

0

1

3

\section{Comportamento Preventivo}

M. Lavo as mãos com frequência e faço uso de álcool gel para mãos e objetos de uso cotidiano.

0
1
2
3

N. Mantenho o distanciamento físico e uso máscara sempre que preciso sair. 0

1

2

O. Evito o consumo exagerado de bebidas alcoólicas.

0

1 
Continuação do Quadro 1 - Apresentação da escala "Perfil do Estilo de Vida Individual” em tempos de distanciamento social, na sua última versão, 2020.

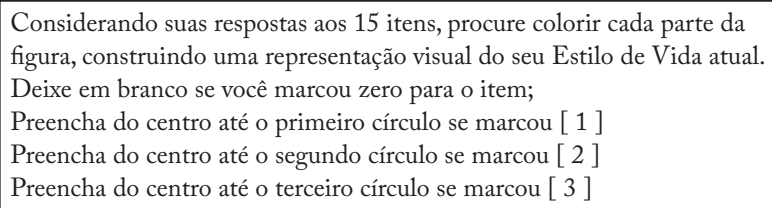

PENTÁCULO DO BEM-ESTAR - EMTEMPOS DE DISTANCIAMENTO SOCIAL

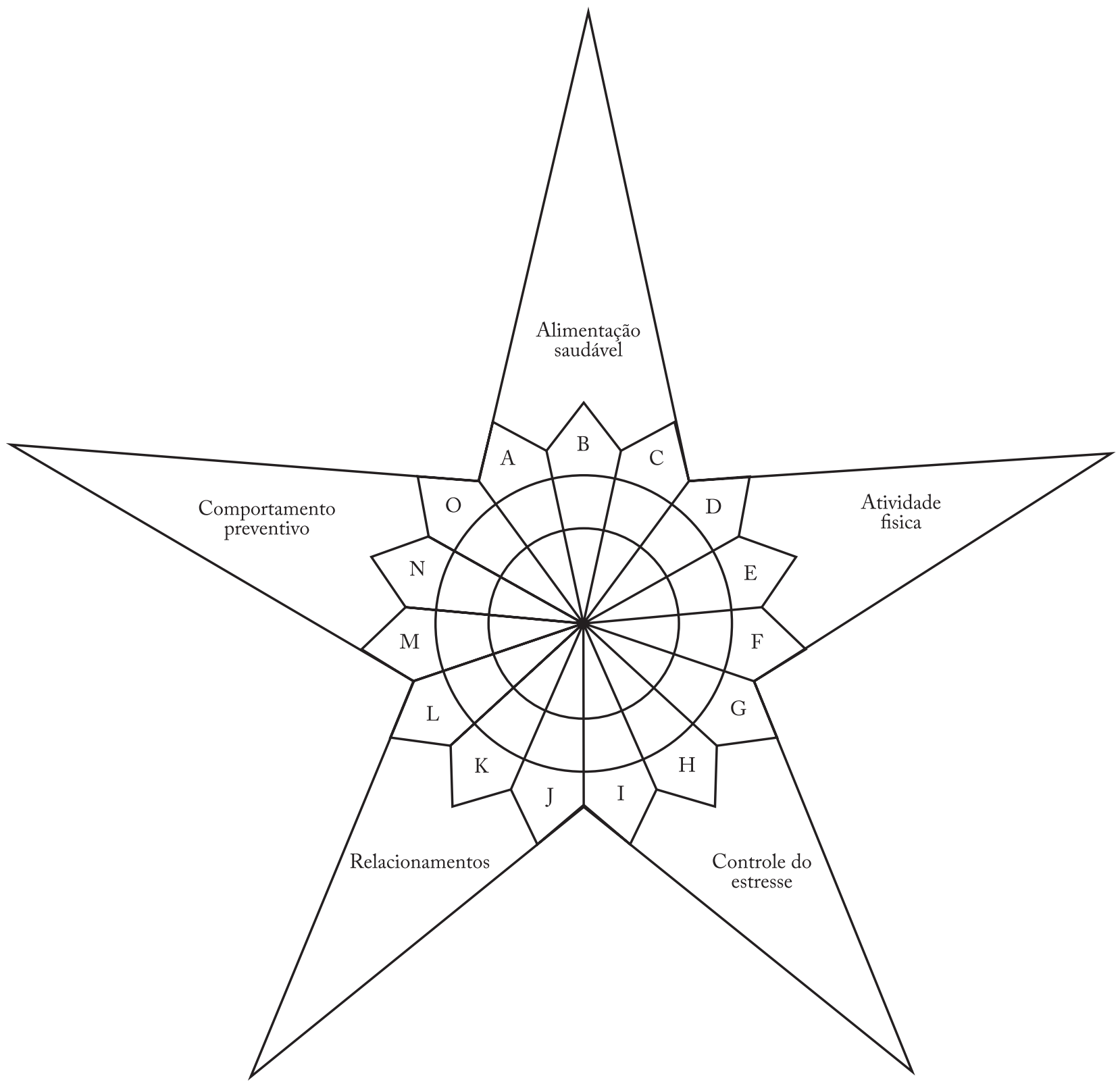

a saúde e qualidade de vida de universitários brasileiros.

Mesmo com a observação de níveis aceitáveis de validade de conteúdo, optou-se em realizar alterações nos itens "F" e "O", tendo sido modificada a redação a partir da sugestão de que o instrumento não abordava o consumo de bebidas alcoólicas como comportamento preventivo e de que itens anteriores tratavam sobre as práticas de atividade física geral, mas não havia ne- 
nhum item que tratasse do deslocamento ativo. Sendo assim, foi alterada a afirmativa do item " $F$ ", de "Aprecio dançar ou assistir vídeos de jogos ou recreação ativos" para "Caminho ou pedalo, mantendo distanciamento seguro, quando preciso ir a lugares próximos"; e do item "O”, de "Após realizar compras, higienizo os produtos e deixo calçados fora de casa" para "Evito o consumo exagerado de bebidas alcoólicas".

Para a estimativa da clareza do instrumento, considerando a participação de voluntários que compreendem a população alvo do instrumento, adultos jovens e de meia-idade, observou-se que a presente escala apresentou valor médio de 78,7\%. Nesta etapa, os itens “D”, "Faço intervalos e me movimento quando passo muito tempo sentado", (clareza: 67,6\%), "G”, "Procuro ocupar a maior parte do meu dia com atividades interessantes" (clareza: 66,2\%) e "L", "Mesmo em isolamento, procuro me manter útil na comunidade" (clareza: 67,6\%) apresentaram valores inferiores a $70 \%$ de clareza. Optou-se pela permanência desses itens com essa estrutura de texto, tendo em vista os valores de validade de conteúdo da etapa anterior, que destacaram pertinência e adequação dessas características em relação ao estilo de vida, bem como a sugestão de adequações dos especialistas.

Quanto a reprodutibilidade, observou-se concordância moderada para 10 itens ("A", "B", "C", "D”, "E”, "F”, “K”, "L", "M", "O”), quatro apresentaram nível fraco/regular (“G”, "H”, "I”, "J”) e em um item a concordância foi forte ("N"). Os itens que apresentaram menores valores de concordância foram referentes ao domínio do controle do estresse e um dos itens do constructo relacionamentos. Outros estudos mostraram escores de concordância maiores que o a presente escala para a auto avaliação do estresse $(\mathrm{k}=0,55)$, relacionamentos $(\mathrm{k}=0,60 \text { e } 47)^{18}$ e ambiente social $(\mathrm{k}=$ $0,61)^{19}$. Possivelmente o contexto social de pandemia, que oscila a cada semana, com a alteração do número de óbitos por COVID-1920,21 e notícias sobre aspectos preventivos e de (não) enfrentamento sociopolítico, pode ter refletido nos níveis de concordância em relação ao controle do estresse, busca por suporte social e atividades no tempo livre para relaxar.

Observou-se concordância forte no item "N", "Mantenho o distanciamento físico e uso máscara sempre que preciso sair". Este resultado reflete a atual estabilidade desse comportamento, o qual foi orientada adoção em todo mundo como principal estratégia para evitar a contaminação e propagação da doença ${ }^{20,22}$.

Níveis de concordância moderadas foram observa- dos em todos os itens dos domínios da atividade física, alimentação saudável, comportamentos preventivos e relacionamentos, corroborando com achados da pesquisa que validou a concordância desta escala para adultos com dor musculoesquelética ${ }^{23}$. Outros estudos demonstraram a qualidade da mensuração dos comportamentos por meio de questionário para o emprego em pesquisas com adultos ${ }^{24-26}$. Por outro lado, ainda se nota o emprego de medidas com ausência de estimativas das capacidades psicométricas ${ }^{27-29}$.

Dentre as limitações deste estudo cita-se a solicitação da análise de clareza considerando a pergunta e a escala de resposta de forma simultânea, fato que pode confundir o respondente, ou seja, perceber a clareza da pergunta diferente da clareza das opções de resposta. Todavia, este critério de análise conjunta foi adotado para não tornar cansativo o processo de análise e assim perder a qualidade nas perguntas finais. Além disso, o intervalo superior a quinze dias para a participação na reprodutibilidade pode ter sido mais sensível em ser influenciado pelas mudanças no estilo de vida em decorrência da pandemia. Optou-se em empregar esse tempo de participação no reteste visando observar o potencial do presente instrumento para o monitoramento do estilo de vida ao longo do tempo. Cita-se também o possível viés de seleção, uma vez que a coleta no formato online limita a participação de grupos populacionais com acesso à internet, especialmente, pessoas de melhor nível socioeconômico, além da falta do registro dos não respondentes ${ }^{8}$.

Por outro lado, torna-se importante caracterizar elementos que congregam a robustez deste estudo. As quatro fases adotadas representam ações positivas empregadas deste estudo, pois desde a concepção do instrumento até a testagem em relação a reprodutibilidade, houve a adesão das medidas em relação ao referencial teórico e a consideração das informações apresentadas por membros do público-alvo. Além disso, a participação de voluntários de diferentes regiões do Brasil, permite ampliar a relevância do processo de validade preliminar quanto aos aspectos socioculturais do público-alvo de destinação deste instrumento. $\mathrm{Ou}$ trossim, nota-se em meio as informações da literatura que esta escala representa uma importante ferramenta de utilização em tempos de distanciamento social e que foi construída para o emprego em brasileiros.

Conclui-se que o nível de validade de conteúdo da escala "Perfil do Estilo de Vida Individual" em tempos de distanciamento social apresentou adequação, perti- 
nência e clareza satisfatórios. Em relação ao nível de concordância, por meio da análise de reprodutibilidade, observou-se concordâncias moderadas para a maioria dos itens. Sugere-se o emprego deste questionário durante a pandemia visando o conhecimento do estilo de vida de adultos para fins educacionais e motivacionais.

\section{Conflito de interesse}

Os autores declaram não haver conflito de interesse.

\section{Contribuição dos autores}

Santos SFS e Sousa TF, participaram da concepção do manuscrito, análise, interpretação dos dados e revisão crítica do conteúdo. Fonseca SA, participou da concepção do manuscrito, redação do manuscrito e revisão crítica do conteúdo. Barros MVG e Nahas $\mathrm{MV}$, participaram da concepção do manuscrito e revisão crítica do conteúdo. Farias GS, participou da redação do manuscrito e revisão crítica do conteúdo.

\section{Agradecimentos}

Os autores agradecem aos colaboradores de todas as universidades envolvidas por viabilizar o contato com os participantes.

\section{Referências}

1. Freitas ARR, Napimoga M, Donalisio MR. Análise da gravidade da pandemia de Covid-19. Epidemiol. Serv. Saúde. 2020;29(2):e2020119.

2. Couto MT, Barbieri CLA, Matos CCSA. Considerações sobre o impacto da COVID-19 na relação indivíduosociedade: da hesitação vacinal ao clamor por uma vacina. Saúde Soc. 2021;31(1):e200450.

3. Malta DC, Szwarcwald CL, Barros MBA, Gomes CS, Machado IE, Souza Júnior PRB, et al. The COVID-19 Pandemic and changes in adult Brazilian lifestyles: a cross-sectional study, 2020. Epidemiol Serv Saúde. 2020;29(4):e2020407.

4. Balanzá-Martínez V, Atienza-Carbonell B, Kapczinski F, Boni RB. Lifestyle behaviours during the COVID-19 - time to connect. Acta Psychiatr Scand. 2020;141(5):399-400.

5. Pereira MD, Oliveira LC, Costa CFT, Bezerra CMO, Pereira MD, Santos CKA. A pandemia de COVID-19, o isolamento social, consequências na saúde mental e estratégias de enfrentamento: uma revisão integrativa. Res Soc Dev. 2020;9(7):e652974548

6. Sousa TF, Santos SFS, Farias GS, Brandão AC, Chaves AO, Mussi FC, et al. Grupos de pesquisa brasileiros com ênfase na pandemia da covid-19. Rev Interfaces Saúde Humanas E Tecnol. 2020;8(3):621-29.

7. Coeli CM, Lima LD, Carvalho MS. Boas práticas na condução e relato de estudos baseados em websurveys. Cad Saúde Pública. 2020;36(7):e00169120.

8. Boni RBD. Websurveys nos tempos de COVID-19. Cad Saúde Pública 2020;36(7):e00155820.

9. Silveira PM, Borgatto AF, Andrade DF, Both J, Nascimento JV. Escala de avaliação do perfil do estilo de vida por meio da teoria da resposta ao item. Rev Educ Física UEM. 2015;26(4):519-27.
10. Both J, Borgatto AF, Nascimento JV, Sonoo CN, Lemos CAF, Nahas MV. Validação da escala "perfil do estilo de vida individual”. Rev Bras Ativ Fis Saúde. 2008;13(1):5-14.

11. Nahas MV, Barros MVG, Francalacci V. O pentáculo do bem-estar - base conceitual para avaliação do estilo de vida de indivíduos ou grupos. Rev Bras Ativ Fis Saúde. 2000;5(2):48-59.

12. Reichenheim ME, Moraes CL. Pillars for assessing validity in epidemiological studies. Rev Bras Epidemiol. 1998;1(2):131-48.

13. Reichenheim ME, Moraes CL. Operacionalização de adaptação transcultural de instrumentos de aferição usados em epidemiologia. Rev Saúde Pública. 2007;41(4):665-73.

14. Nahas MV. Atividade física, saúde e qualidade de vida. $7^{\mathrm{a}} \mathrm{ed}$. Florianópolis: Ed. do Autor, 2017.

15. Crestani AH, Moraes AB, Souza APR. Validação de conteúdo: clareza/pertinência, fidedignidade e consistência interna de sinais enunciativos de aquisição da linguagem. CoDAS[online]. 2017;29(4):e20160180.

16. Landis JR, Koch GG. The measurement of observer agreement for categorical data. Biometrics. 1977;33(1):159-74.

17. Santos SG. Métodos e Técnicas de Pesquisa Quantitativa Aplicada à Educação Física. 1º ed. Florianópolis: Tribo ilha, 2011.

18. Sousa TF, Fonseca SA, José HPM, Nahas MV. Validade e reprodutibilidade do questionário Indicadores de Saúde e Qualidade de Vida de Acadêmicos (Isaq-A). Arq Ciênc Esporte. 2013;1(1):21-30.

19. Nahas MV, Rabacow FM, Pereira SV, Borgatto AF. Reprodutibilidade de uma escala para avaliar a percepção dos trabalhadores quanto ao ambiente e às condições de trabalho. Rev Bras Saúde Ocupacional. 2009;34(120):179-83.

20. Acter T, Uddin N, Das J, Akhter A, Choudhury TR, Kim S. Evolution of severe acute respiratory syndrome coronavirus 2 (SARS-CoV-2) as coronavirus disease 2019 (COVID-19) pandemic: A global health emergency. Sci Total Environ. 2020;730:138996.

21. Souza CDF, Paiva JPS, Leal TC, Silva LF, Santos LG, Souza CDF, et al. Evolução espaçotemporal da letalidade por COVID-19 no Brasil, 2020. J Bras Pneumol. 2020;46(4):e20200208.

22. Aquino EML, Silveira IH, Pescarini JM, Aquino R, SouzaFilho JA, Rocha AS, et al. Social distancing measures to control the COVID-19 pandemic: potential impacts and challenges in Brazil. Ciênc saúde coletiva. 2020;25(Suppl1):2423-46.

23. Modenutte GS, Hotta JMGH, Oliveira AS. Confiabilidade intra e interexaminador do questionário Perfil do Estilo de Vida Individual (PEVI) em indivíduos com dor musculoesquelética. Fisioter. Pesqui. 2019; 26 (1):71-7.

24. Molina M del CB, Benseñor IM, Cardoso LO, Velasquez-Melendez G, Drehmer M, Pereira TSS, et al. Reprodutibilidade e validade relativa do Questionário de Frequência Alimentar do ELSA-Brasil. Cad Saúde Pública. 2013;29(2):379-89.

25. Moreira AD, Claro RM, Felisbino-Mendes MS, VelasquezMelendez G. Validade e reprodutibilidade de inquérito telefônico de atividade física no Brasil. Rev Bras Epidemiol. 2017;20(1):136-46.

26. Chopra S, Ranjan P, Malhotra A, Sahu A, Dwivedi SN, Baitha U, et al. Development and validation of a questionnaire to evaluate the impact of COVID-19 on lifestyle-related behaviors: eating habits, activity and sleep behavior. Public Health Nutr. 2021;24(6):1275-190. 
27. Dumith SC. Physical activity in Brazil: a systematic review. Cad Saúde Pública. 2009;25(Suppl 1):S415-26.

28. Hallal PC, Dumith SC, Bastos JP, Reichert FF, Siqueira FV, Azevedo MR. Evolução da pesquisa epidemiológica em atividade física no Brasil: revisão sistemática. Rev Saúde Pública. 2007;41(3):453-60.
29. Silva TA, Vasconcelos SML. Procedimentos metodológicos empregados em questionários de frequência alimentar elaborados no Brasil: uma revisão sistemática. Rev Nutr. 2012;25(6):785-97.

Recebido: 24/02/2021

Aprovado: 21/06/2021

\section{Como citar este artigo:}

Santos SFS, Sousa TF, Fonseca SA, Barros MVG, Farias GS, Nahas MV. Validade Preliminar da Escala Perfil do Estilo de Vida Individual para uso online em tempos de distanciamento social. Rev Bras Ativ Fís Saúde. 2021;26:e0210. DOI: 10.12820/rbafs.26e0210 